\title{
Result Evaluation of Contention Based Attribute Prediction Model in Information Security
}

\author{
Neha Mourya ${ }^{1}$, Margi Patel ${ }^{2}$ \\ ${ }^{1}$ Research Scholar, Computer Science and Engineering Department, Oriental University, Indore \\ ${ }^{2}$ Assistant Professor, Computer Science and Engineering Department, Indore Institute of Science \& Technology, Indore
}

\begin{abstract}
To make data in incongruous structure uncountable approaches are admonished by investigates. Basically the framework to make data indistinct structure is named as encryption or cryptography. Cryptography or encryption computations act a basic part in data security. Disseminated figuring gives exceptionally versatile and more tried and true stockpiling on untouchable trusted servers. It is the blend of different models like grid preparing, dispersed enlisting, utility figuring, and autonomic figuring et cetera. Its sensible pay-per-use utility model results in a reducing of the cost of sending of the same handling resources locally. The key stress over dispersed registering is data outsourcing to a cloud which is the stockpiling of essential information related to client's structure in pariah servers at appropriated territories. It is legitimate for any class of usages that obliges data to be kept away and spread to various customers. Arrangement a deniable $\mathrm{CB}-\mathrm{ABE}$ arrangement with composite solicitation bilinear social affairs for building audit free circulated stockpiling organizations. Composite solicitation bilinear get-togethers have two engaging properties, specifically suspecting and wiping out. We make use of the dropping property for building an anticipated circumstance; on the other hand, Freeman moreover pointed out the basic issue of computational cost with regards to the composite solicitation bilinear social affair. [1]
\end{abstract}

Keywords: Cryptography System, Distributed Network, Information Security system, Attribute Based Encryption (ABE), Contention Based (CB-ABE)

\section{Introduction}

Attribute based encryption (ABE) is an open key based oneto-various encryption that grants customers to encode and disentangle data in perspective of customer properties. A promising use of $\mathrm{ABE}$ is versatile access control of encoded data set away in the cloud, using access polices and acknowledged attributes associated for private keys and cipher texts. One of the main capability inconveniences of the current $\mathrm{ABE}$ arrangements is that translating incorporates excessive mixing operations and the amount of such operations creates with the disperse nature of the passageway approach. Starting late, Green et al. proposed an ABE system with outsourced unscrambling that, all things considered, wipes out the disentangling overhead for customers. In such a system, a customer gives an entrusted server, say a cloud organization supplier, with a change key that allows the cloud to unravel any $\mathrm{ABE}$ cipher text satisfied by that customer's qualities or access approach into a fundamental cipher text, and it just realizes somewhat computational overhead for the customer to recover the plaintext from the changed cipher text. Security of an ABE system with outsourced translating ensures that an enemy (checking a dangerous cloud) won't have the ability to learn anything about the encoded message; then again, it doesn't guarantee the precision of the change done by the cloud. In this paper, we consider another need of ABE with outsourced deciphering: undeniable status. Calmly, undeniable status guarantees that a customer can capably check if the change is done viably. We give the formal model of $\mathrm{ABE}$ with obvious outsourced disentangling and propose a strong arrangement. We show that our new arrangement is both secure and apparent, without relying upon unpredictable prophets. Finally, we exhibit an execution of our arrangement and eventual outcome of execution estimations, which demonstrates a basic diminishment on figuring resources constrained on customers. [2]

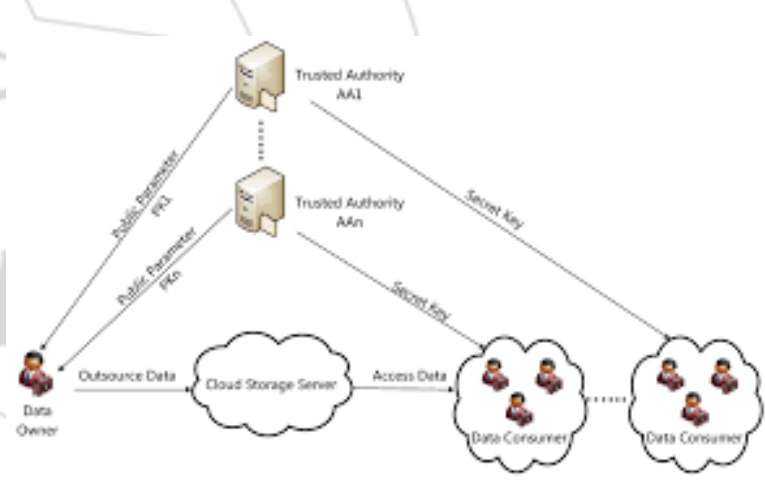

Figure 1: ABE Process

Quality based encryption is similarly called as behavior based encryption (BBE). In this the cipher texts and customer keys are associated with techniques that delineate the customer that is allowed to get to the mixed information. Specifically, in Key-Policy ABE (KP-ABE) cipher texts are mixed with a course of action of characteristics and each customer's puzzle key is associated with a methodology portraying which figure compositions customer can unravel. Such a system is a predicate over the course of action of qualities, ordinarily characterized as a Boolean comparison. [3] 


\section{International Journal of Science and Research (IJSR) \\ ISSN (Online): 2319-7064}

Index Copernicus Value (2013): 6.14 | Impact Factor (2015): 6.391

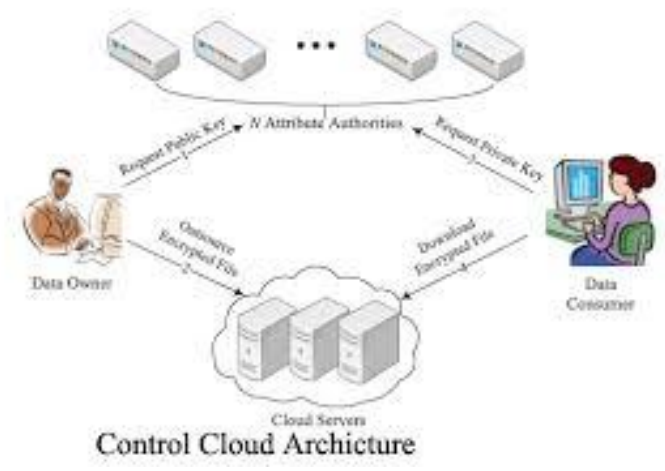

Figure 2: Authentication Process

\section{Literature Survey}

Cipher texts Figure writings approach property based encryption is an arrangement that gives a trademark way to deal with secluded the accreditations from the passageway game plan and acutely go along with them at a later stage to give secure access to guaranteed data. In most $\mathrm{ABE}$ arrangements the range of the figure writings is extremely immense and is of the solicitation of the amount of properties. In this work we show our philosophy for a multilevel edge trademark based encryption which is free of the amount of properties.

To deal with the previously stated destinations of access control and better encryption standard a champion amongst the most promising philosophy can be used named as trademark based encryption through figure message just systems. In this arrangement, customers have sets of characteristics (and relating secret trademark keys) that depict certain properties. [4]

Figure writings are mixed by access control methodology, characterized as a Boolean formula over the qualities. The advancement ensures that just customers whose qualities satisfy the passageway control methodology can unscramble the figure writings with their puzzle property keys [5]

The advancement is required to satisfy a scheme resistance property: It must be tremendous for a couple of customers to pool their characteristic keys such that they can disentangle a figure writing which they would not have the ability to unscramble independently. [6]

There is such an assortment of other change based arrangements open like HNT Transformation [7], Bayes Network and HMM [8] and hop by bob framework for acceptance [9]. These above security and affirmation instrument can moreover be associated in various spaces like used as a piece of [10].

\section{Problem Statement}

It proposed an $\mathrm{ABE}$ structure with outsourced translating that, all things considered, discards the unscrambling overhead for customers. In such a system, a customer gives an entrusted server, say a cloud organization supplier, with a change key that allows the cloud to disentangle any ABE cipher text satisfied by that customer's qualities or access procedure into a direct cipher text, and it just gains somewhat computational overhead for the customer to recover the plaintext from the changed cipher text. One of the guideline efficiency disservices of the most existing $\mathrm{ABE}$ arrangements is that unscrambling is expensive for resource compelled devices in view of mixing operations, and the amount of coordinating operations required to disentangle a cipher text creates with the multifaceted way of the passage game plan. To the detriment of security, simply showed in a weak model (i.e., particular security), there exist a couple of expressive $\mathrm{ABE}$ arranges where the unscrambling estimation just requires a relentless number of mixing computations. In this paper, we first conform the principal model of $\mathrm{ABE}$ with outsourced interpreting in existing system to consider assurance of the progressions. Resulting to portraying the formal importance of conspicuousness, we propose another $\mathrm{ABE}$ exhibit and in perspective of this new model build up a strong ABE arrangement with certain outsourced unscrambling. Our arrangement does not rely on upon sporadic prophets.

\section{Proposed Solution}

Information Data caution from unapproved customer's disclosure at right on time stages arranges the customer properties and gives access in consent to that. On-interest disavowal ought to be conceivable by completing customer properties and gives fine grained data access approach. Form access control from unlawful customer exceptional in connection to data holder get-together can be given to redesign further security however revocable characteristic based encryption. Adaptably Scalable and fit usability based ranges to offer the customer separation of work locale and data designation. It should handle all the confounded issues to make the approach more suitable. Proposed methodology of CTPM can be used for both CP-ABE and KP-ABE and particularly expected for data stockpiling. Security Analysis of the Proposed System

(i)Fine-grainedness of Access Control: Resulting to focusing on the diverse available parts and functionalities of existing structures there are some new necessities which is making for further security improvements. For instance, in existing system data proprietor is not having any control over methodology delineation and pertinence of access structure. Subsequently the new system can describe and actualize expressive and versatile access structure for each customer. Specifically, the passage structure of each customer is portrayed as a justification mathematical statement over data record qualities, and can address any pined for data archive set.

(ii)Data thoughtfulness: In the present system the data order is only a strategy matter of encryption gages and thusly some standard approachs is used which is open with attackers also. Along these lines is such case the security can be exchanged off. Thusly some novel an arrangement ought to be prescribed which manages the information plan and gives the passage to the data as showed by the customer chronicled activity and after their inferable parts affirmation just. The 


\section{International Journal of Science and Research (IJSR) \\ ISSN (Online): 2319-7064 \\ Index Copernicus Value (2013): 6.14 | Impact Factor (2015): 6.391}

data can be simply known not customers whose characteristic qualities may organize with portrayed parameters.

(iii)User Access Privilege judiciousness: Customer advantage needs to gage nearby their credits for right data to right customers. Infers each data access must take after the properties of data restriction nonattendance of which prompts off course data appear.

\section{Result Analysis}

Into execute them gear and programming resources are required, consequently the once-over of fancied resources and their specific points of interest are given in this area. In addition of that, this section fuses the re-establishment parameters and executed framework circumstances. This portion of the file gives cognizance of entertainment and its determinations in unpretentious component. Already discussed part examination and blueprint of needed system is done, yet

Key Generation Policy \& ABE

Input Test Set User 1 (Isolation \& Encryption using ABE)

Environment Variable Conditions:

File Size between $10 \mathrm{~kb}$ to $100 \mathrm{~kb}$

Key 1 (Credential \& Timestamp) $=$

(harsh)XOR(123456789)XOR(Current Timestamp)

Key $2($ Login Failed Attempt $($ LFA $))=2$

Key 3 (Type of File Access $($ TFA $))=($ doc,txt,xls,jpeg,bmp)

ID Values XORed

Key 4 (Services $)=($ Chat, Calculator, Contact, File sharing, Map, Data Storage) ID Values XORed
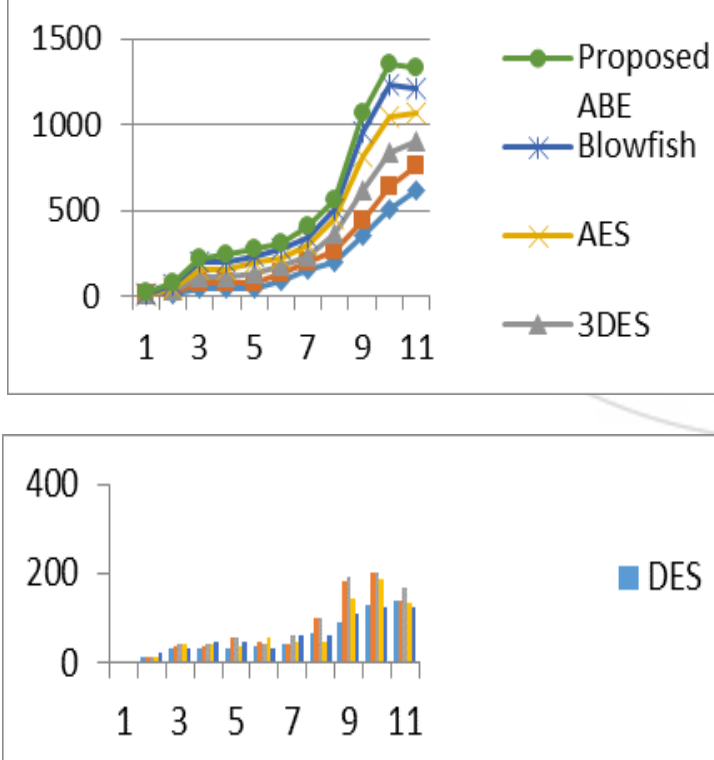

Table 1: Graph with respect to speed and time

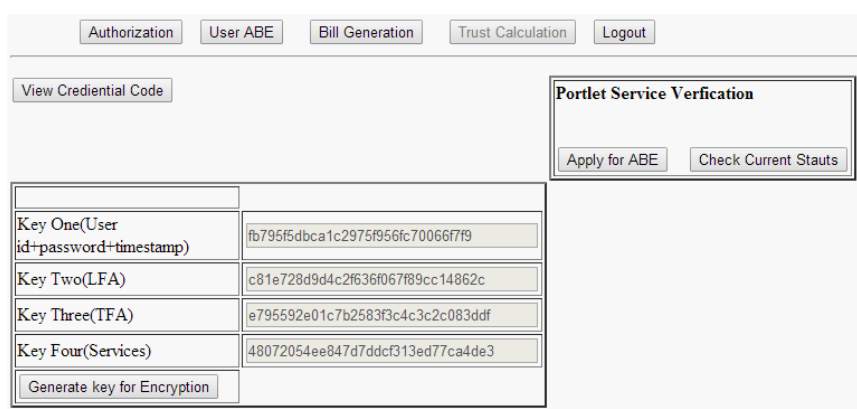

Figure 2: Key Generated Process

The page at localhost:9595 says:

Your Bill

Chat : 0.0

Calculator : 0.0

Calender : 0.0

Contacts : 0.3611111

Data Storage : 0.9722222

File Sharing : 0.0

Messaging : 0.027777778

Map : 0.06666667

Video Calling : 0.0

Total : 1.4277778

OK

Figure 3: Details of process used

\section{Conclusions}

The proposed key based arrangement has another property that we can controlled customer end security which is not known not present (to the best of our knowledge) in any of the past key based trademark mark arranges. This is a component that would allow the customer key to control their mystery paying little heed to the likelihood that it will perform on any framework whether is not controlled by them or not. Allow us to say Alice is denoting a record which needs a key to give security, and she has sufficient credits to satisfy the result.

Data is noteworthy assets for client considering singular, business, social and wellbeing information often sharable separate to time and need. The nonattendance of get ready time and limit cut-off or extra resources cost data set away at third place known as cloud suppliers instead of client use its own particular resources. Regardless, there have been wide insurance stresses as data could be displayed to those third place servers and to unapproved parties. To ensure the client control over access to its own information's, it is a promising strategy to make data obfuscated and non-interpretable structure. [11] 


\section{References}

[1] John Bethencourt, Amit Sahai \& Brent Waters, "Ciphertext-Policy Attribute-Based Encryption", in NSF CNS-0524252 US Army Research, in 2009.

[2] Kan Yang, Zhen Liu, Zhenfu Cao, Xiaohua Jia, Duncan S. Wong \& Kui Ren, "TAAC: Temporal Attribute-based Access Control for Multi-Authority Cloud Storage Systems" in University at Buffalo, 2011.

[3] Yan Zhu, Hongxin Hu, Gail-Joon Ahn, Xiaorui Gong \& Shimin Chen, "POSTER: Temporal Attribute-Based Encryption in Clouds" in ACM CCS 11, ISSN: 978-14503-0948-6/11/10, Dec 2011.

[4] Sushmita Ruj, Amiya Nayak \& Ivan Stojmenovic, "DACC: Distributed Access Control in Clouds" in IEEE TrustCom-11/IEEE ICESS-11, ISSN 978-0-7695-46001/11, 2011.

[5] Amit Sahai \& Hakan Seyalioglu, "Dynamic Credentials and Ciphertext Delegation for Attribute-Based Encryption" in DARPA N11AP20006, University of Texas, Aug 2012.

[6] Changji Wang \& Jianfa Luo, "An Efficient Key-Policy Attribute-Based Encryption Scheme with Constant Ciphertext Length" in Mathematical Problems in Engineering Volume 19, Article ID 810969, 2013.

[7] Nishant Doshi \& Devesh Jinwala, "Updating Attribute in CP-ABE: A New Approach" in IJCA ICDCIT, ISSN 0975 - 8887, 2013.

[8] Neena Antony \& A. Alfred Raja Melvin, “An Efficient Approach for Flexible and Scalable Access Control Through HASBE" in IJCSMR Vol 2 Issue 4, ISSN 2278-733X, April 2013.

[9] Sunitha Muppa, R. Lakshman Naik \& Chalapathi Valupula, "Secure Scheme of Data Protection in Cloud Computing" in IJCST Vol. 3, Issue 1, ISSN: 0976-8491, Mar 2012.

[10] Shilpa Elsa Abraham, "Distributed Attribute Based Encryption for Patient Health Record Security under Clouds" in IJCTT, Vol 4 Issue 3, 2013.

[11] Anup R. Nimje, V. T. Gaikwad \& H. N. Datir, "Attribute-Based Encryption Techniques in Cloud Computing Security: An Overview" in IJCTT, Vol 4 Issue 3, 2013.

\section{Author Profile}

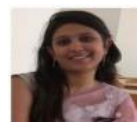

Neha Mourya received the Bachelors of Engineering in Information Technology from Shri Dadaji Institute of Technology \& Science Khandwa (M. P.), India and she is a Research Scholar at Oriental University, Indore, India. Her major research areas are networking, distributed computing etc.

Margi Patel received the Bachelors of Engineering in Information Technology from J.I.T Borawan, Khargone and Masters of Engineering degrees in Computer \& Science Engineering from IET DAVV University, Indore. Her Masters is in Software Engineering and working as an Assistant Professor at Indore Institute of Science \& Technology, Indore. Her major research areas are distributed computing, MANET, networking, etc. 